\title{
Mode-Locking with Slow and Fast Saturable Absorbers-What's the Difference?
}

\author{
Franz X. Kärtner, Juerg Aus der Au, and Ursula Keller, Member, IEEE
}

(Invited Paper)

\begin{abstract}
We investigate the differences in the dynamics of lasers mode-locked by fast and slow saturable absorbers. Slow saturable absorbers can already generate almost transform limited pulses much shorter than the recovery time of the absorber. If soliton-like pulse shaping is present in addition the pulses can be further compressed below the resulting net gain window until either the continuum breaks through or the pulses break up into multiple pulses, which sets a limit to the shortest pulsewidth achievable. Given a certain amount of saturable absorption, a comparison is made that results in an estimate for the shortest pulse achievable for a solitary laser stabilized by a fast or a slow saturable absorber. The theoretical results are compared with experiments.
\end{abstract}

Index Terms-Multiple pulsing, pulsed lasers, semiconductor saturable absorbers, solid lasers.

\section{INTRODUCTION}

$\mathbf{F}$ OR A LONG TIME, it was believed that the use of a fast saturable absorber is absolutely necessary to modelock a solid-state laser, because mode-locking relies on a short net gain window that only supports the pulse and discriminates against the noise that might grow outside the pulse. However, over the last several years many solid-state lasers have been mode-locked using semiconductor saturable absorbers mirrors SESAM's [1]-[3], that have very often much longer recovery times than the shortest pulses generated from the given laser. One reason for this behavior has been traced back to the soliton-like pulse formation, i.e., negative group delay dispersion (GDD) and self-phase modulation (SPM), occuring in femtosecond solid-state lasers [4]-[6]. The solitonlike pulse formation leads to stable pulsing even in the presence of a considerable open net gain window following the pulse. The pulse is not any longer shaped dominantly by the saturable absorber, but the absorber is still essential for pulse stability. Note, this regime of operation is significantly different from what has been discussed before in the context of the CPM-dye lasers, where the interplay between loss and gain saturation always leads to a short net gain window in time [7]-[10]. The same is very much true for the fast saturable absorber, where the soliton-like pulse shaping leads to pulses about a factor of two shorter than without soliton-like pulse

Manuscript received December 4, 1997. This work is supported by the Swiss National Science Foundation.

The authors are with the Ultrafast Laser Physics Laboratory, Institute of Quantum Electronics, Swiss Federal Institute of Technology, ETH Hönggerberg-HPT, CH-8093 Zürich, Switzerland.

Publisher Item Identifier S 1077-260X(98)03844-1. shaping [11]. The open net gain windows that form in these cases are only about one pulsewidth long.

In this paper, we further discuss the difference in the dynamics of solid-state lasers mode-locked by fast or slow saturable absorbers with and without soliton-like pulse shaping. We show that a slow saturable absorber can generate pulses much shorter than the recovery time of the absorber already without soliton-like pulse shaping. Of course, the soliton-like pulse shaping helps to compress the pulse even further until the continuum breaks through as discussed in [4] and [5] or the pulse breaks up into multiple pulses, as has been shown experimentally in [12] and [13]. We present simple relations, that indicate when the single pulse per cavity round-trip will break up into longer multiple pulses due to the solitonlike pulse shaping. The breakup criterion is compared with experimental results observed recently with a Nd:glass laser. The breakup into multiple pulses sets limits to the shortest pulse achievable with a given saturable absorber even if the laser is stable against continuum breakthrough. The criterion for pulse breakup can be applied to lasers mode-locked by fast as well as slow saturable absorbers. The analysis shows that the laser is most stable against multiple pulse breakup, if the absorber is about three times saturated (i.e., the intracavity pulse energy is about three times the saturation energy of the absorber) for the case where the saturable absorption is only a small contribution to the overall cavity losses. The case of a slow saturable absorber is interesting for two reasons. First of all, every fast saturable absorber becomes slow if the pulsewidth is short enough. Second, in many cases the dynamics of semiconductor saturable absorbers is well described by a simple slow saturable absorber. Such absorbers have been used very successfully to mode-lock a variety of solidstate lasers down to the 10-fs regime [14]-[16]. The question arises, how effective is a slow saturable absorber in modelocking in comparison with a fast saturable absorber with equal strength, i.e., modulation depth. Of course, one could always generate a shorter pulse by increasing the modulation depth. But usually every saturable absorber introduces undesired nonsaturable losses which increase with larger modulation depth and degrade the performance of the laser. Thus a given saturable absorption should be utilized as efficiently as possible for the generation of short pulses. A brief conclusion is that we can obtain with the same modulation depth almost equally short pulses with a fast saturable absorber and a slow saturable absorber, if the absorber recovery time is not more 
than a factor of ten slower than the pulsewidth for optimum saturation. However, the fast saturable absorber is more stable against multiple pulse breakup in case of oversaturation of the absorber.

The discussion in this paper is based on the assumption of small linear and nonlinear pulse shaping effects occuring within each intracavity element per round-trip, such that we can apply Haus' master equation [17]. However, most of the results can be carried over, at least qualitatively, to lasers where the pulse shaping in each element leads already to substantial changes on the laser dynamics. This is most pronounced in the 10-fs and sub-10-fs Ti:sapphire lasers [18]-[20]. Numerical simulations show that the influence of the discrete action of the individual elements for a given situation, e.g., overdriven SPM within one round-trip, can be considered as increased losses for shorter pulses similar to a finite-gain bandwidth. Therefore, overdriven SPM should be avoided. For example, the stretched-pulse mode-locking technique in femtosecond fiber lasers strongly reduces the SPM [21], [22]. For this reason, we stay in our discussion within the master equation approach.

\section{BASIC MODEL}

\section{A. Analytic Approach}

We start from the well established model for the pulse shaping process in a solid-state laser, where we assume, that the linear and nonlinear changes in the pulse within each round-trip in the laser cavity stays small. Then the average dynamics of the pulse can be described by Haus' master equation:

$$
\begin{aligned}
T_{R} \frac{\partial}{\partial T} A(T, t)= & \left(-i D \frac{\partial^{2}}{\partial t^{2}}+i \delta|A|^{2}\right) A \\
& +\left(g-l+D_{g, f} \frac{\partial^{2}}{\partial t^{2}}-q(T, t)\right) A
\end{aligned}
$$

Here $A(T, t)$ is the slowly varying field envelope, $T_{R}$ the cavity round-trip time, $D$ the intracavity GDD, $D_{g, f}=$ $g / \Omega_{g}^{2}+1 / \Omega_{f}^{2}$ the gain and possible filter dispersion, $\delta$ the SPM coefficient, $l$ the round-trip losses, and $g$ the saturated gain. $\Omega_{g}$ is the half-width at half-maximum (HWHM) gain bandwidth and $\Omega_{f}$ the filter HWHM bandwidth. $q(t)$ denotes the saturable absorption coefficient. The SPM coefficient is given by the nonlinear refractive index $n_{2}$ of the laser crystal, the effective area $A_{\text {eff }}$ of the laser mode in the crystal and the length of the crystal $\ell$ according to $\delta=\left(2 \pi n_{2} / \lambda_{o} A_{\text {eff }}\right) \ell$. For solid-state lasers the gain-cross section is very small, such that for typical intracavity pulse energies the gain saturation due to a single pulse is negligible. Then the gain saturates only over many pulses, i.e., with the average power. In the following, we are not concerned with instabilities due to $Q$-switching which is an interesting subject on its own [23], [24]. Thus, we always assume that the gain is saturated to its stationary value corresponding to the average power $P(T)=E_{P}(T) / T_{R}$

$$
g(T)=\frac{g_{0}}{1+\frac{E_{P}(T)}{P_{L} T_{R}}}
$$

where $g_{0}$ is the small-signal gain, $E_{P}(T)=\int_{-\infty}^{+\infty}|A(T, t)|^{2} d t$ gives the pulse energy, and $P_{L}$ is the saturation power of the gain medium. The saturable absorber can be fast or slow. In case of a fast absorber, the response within each round-trip on the instantaneous pulse power is given by

$$
q(T, t)=\frac{q_{0}}{1+\frac{|A(T, t)|^{2}}{P_{A}}}
$$

where $q_{0}$ is the nonsaturated but saturable loss of the absorber and $P_{A}$ is the saturation power of the saturable absorber. In case of a slow saturable absorber, i.e., the recovery time $\tau_{A}$ of the saturable absorber is comparable or even longer than the final pulsewidth. In this case, the absorber is assumed to saturate according to the following:

$$
\frac{d q(T, t)}{d t}=-\frac{q-q_{0}}{\tau_{A}}-q \frac{|A(T, t)|^{2}}{E_{A}}
$$

Note, in the limit of a very short absorber recovery time the solution of (4) approaches (3). We always assume that the absorber recovers completely between two consecutive cavity round-trips of the pulse. $E_{A}$ is the saturation energy of the absorber. Then the master equation (1) complemented with the absorber (4) determines our laser model completely.

\section{B. Numerical Approach}

Only for a few cases the steady state solution according to (4) can be found analytically. One case is the ideal fast saturable absorber which will be discussed in the next section. In general the steady state solution will be found numerically by simple simulation of the laser dynamics according to (1) and (4) until a steady state is reached within the available computation time. However, in many cases there is no steady state solution anymore, then we will simply stop after 20000 round-trips and discuss the final result. In the real laser, the dispersion and the SPM is lumped in the individual elements and does not occur distributed in the average sense as described by (1). Therefore, we simulate (1) by the standard split-step-Fourier-transform method [25]. The linear effects are treated in the frequency domain in one step and the nonlinear effects are treated in the time domain in one step. The simulation uses the laser and absorber parameters (Table I) observed in the $\mathrm{Nd}$ :glass laser experiments presented in [12]. The small-signal gain is varied from 0 to 0.2 in steps of 0.01 . The resulting pulse intensities achieved after 20000 cavity round-trips for different parameter settings will be computed. Note, the maximum amount of saturable absorption is half a percent, see Table I, therefore, it produces only a small change in the pulse shape within each round-trip. For long pulses with low peak power also the effects due to GDD and SPM are small, so that the numerical solution will mimic the solution of the average model (1). However, when the pulses get shorter the lumped action of the GDD and SPM can grow and the simulation will deviate from the average model. We will discuss effects due to the discrete action of SPM and GDD in Section V. 
TABLE I

Parameter Values Used in the Simulations

\begin{tabular}{c|c}
\hline Parameter & Value \\
\hline \hline$l$ & 0.01 \\
\hline$g_{o}$ & $0-0.2$ \\
\hline$\Omega_{g}$ & $2 \pi \cdot 4 \mathrm{THz}$ \\
\hline$P_{L}$ & $0.8 \mathrm{~W}$ \\
\hline$E_{A}$ & $17 \mathrm{~nJ}$ \\
\hline$\tau_{A}$ & $50 \mathrm{fs}-10 \mathrm{ps}$ \\
\hline$q_{0}$ & 0.005 \\
\hline$D$ & $-75 \mathrm{fs}$ \\
\hline$\delta$ & $0.2 / \mathrm{MW}$ \\
\hline
\end{tabular}

\section{Pulse Formation Without GDD AND SPM}

\section{A. Mode-Locking with an Ideal Fast Saturable Absorber}

In the following, we consider different cases: First, the well studied fast saturable absorber model [17]. We assume no dispersion and refractive index changes and that the absorber response time is much faster than the resulting pulsewidth. Then the absorber saturates with the ratio between the instantaneous power of the pulse and the saturation power $P_{A}=E_{A} / \tau_{A}$ of the absorber according to (3). If the peak intensity of the pulse is much smaller than the saturation intensity of the absorber, we can linearize (3) and obtain

$$
l_{\mathrm{tot}}=l+q_{0}-\gamma|A(T, t)|^{2}
$$

where

$$
\gamma=\frac{q_{0}}{P_{A}}
$$

For the linearized absorber model, the steady state solution of the master equation (1) can be solved analytically and a sech-pulse solution is obtained [17]

$$
A(T, t) \rightarrow A_{S}(t)=A_{0} \operatorname{sech}\left(\frac{t}{\tau}\right)
$$

where $\tau_{F W H M}=1.76 \cdot \tau$. Using the solution (6) with (5) in (1), results in a saturated gain for the sech-pulse

$$
g=l+\frac{D_{g, f}}{3 \tau^{2}}+q_{S}\left(E_{P}\right)
$$

where $E_{P}=2\left|A_{0}\right|^{2} \tau$ is the energy of the sech-pulse and $q_{S}\left(E_{P}\right)$ is the saturable amplitude loss encountered by the sech-pulse within one round-trip

$$
q_{S}\left(E_{P}\right)=\frac{1}{2 \tau} \int_{-\infty}^{+\infty} \operatorname{sech}^{2}\left(\frac{t}{\tau}\right) q(t) d t .
$$

This is the residual loss the pulse sees during saturation of the absorber and, therefore, $q(t)$ is here the response calculated from (4) with the solution (6).

The steady-state pulse energy and the pulsewidth $\tau$ of the sech-pulse are related to each other because the pulse

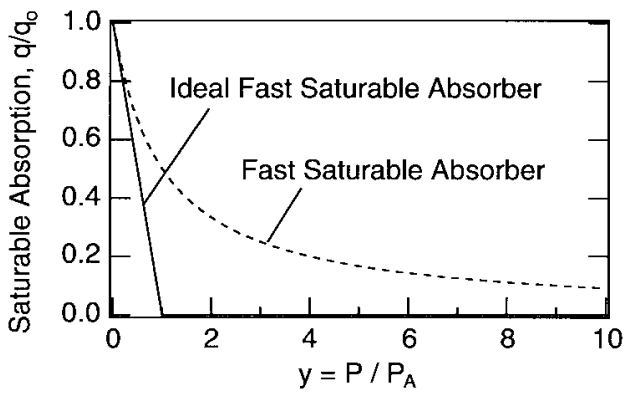

Fig. 1. (---) Saturation characteristic of the fast saturable absorber and (-) the piecewise-linear characteristic of the ideal fast saturable absorber.

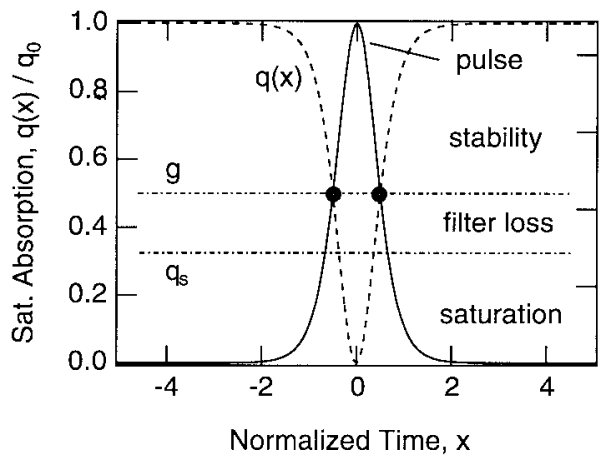

Fig. 2. Normalized loss and gain dynamics for a sech $(x)$-pulse of a laser mode-locked by a ideal fast saturable absorber in the regime of linear and full saturation.

broadening due to gain filtering is balanced by the pulse shortening due to the saturable absorber in each round-trip [17]

$$
\frac{D_{g, f}}{\tau^{2}}=\frac{1}{2} \gamma\left|A_{0}\right|^{2} \quad \text { or } \quad \tau=\frac{4 D_{g, f}}{\gamma E_{p}} .
$$

For the case of the linearized fast saturable absorber (5), we obtain

$$
q_{S}\left(E_{P}\right)=q_{0}-\frac{\gamma E_{P}}{3 \tau}=q_{0}-\frac{4 D_{g, f}}{3 \tau^{2}} .
$$

The resulting gain loss balance (7) is then given by

$$
g=l+q_{0}-\frac{D_{g, f}}{\tau^{2}} .
$$

Let us assume, that we exploit the absorber as much as possible, to create the shortest pulse, that is the absorber is completely saturated at the peak of the pulse, i.e., $q_{0}=\gamma\left|A_{0}\right|^{2}$, but we still want to use the linearized saturable absorber characteristic, (Fig. 1). Thus, we approximate the saturable absorber characteristic by a piecewise linear function. Fig. 2 shows the time dependent saturable absorption as a function of the normalized time $x=t / \tau$ together with the position of the saturated gain level according to (9)-(11) and the pulse power, for this case. Equations (9) and (11) show that the saturated gain level is exactly placed at the full-width at halfmaximum (FWHM) points of the absorber modulation which opens and closes the net gain window, that supports the pulse, immediately in front and after the pulse. Then (9) and (10) show, that the pulse still experiences one third of the saturable 


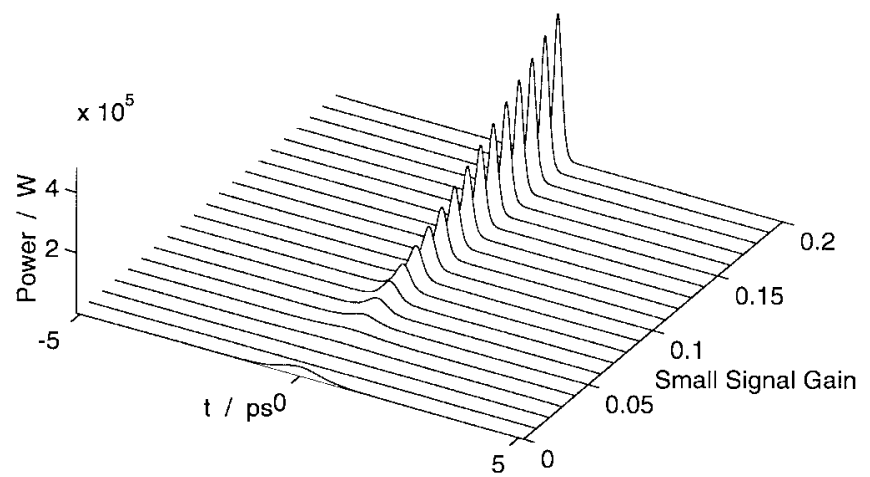

Fig. 3. Each trace shows the stationary pulse intensity profile obtained after 20000 cavity round-trips with an absorber response time $\tau_{A}=50 \mathrm{fs}$ for the different values of the small-signal gain indicated on the left. The simulations are always started with a 1-ps initial pulse shown as the first trace.

loss during saturation of the absorber. Only one sixth of the total saturable absorption is used to overcome the filter losses of the pulse due to the finite gain bandwidth. The remaining one half of the available saturable absorption is reserved for pulse stability, i.e., the net gain window is kept as short as the FWHM of the amplitude profile. Therefore, instabilities that might grow outside the pulse are suppressed. From (9) follows, that for full saturation of the linearized fast saturable absorber, i.e.,

$$
2 \frac{D_{g, f}}{\tau^{2}}=\gamma\left|A_{0}\right|^{2}=q_{0}
$$

the minimum FWHM pulsewidth that can be achieved given a certain amount of saturable absorption results in

$$
\tau_{\mathrm{FWHM}, \mathrm{FSA}}=1.76 \cdot \sqrt{2 D_{g} / q_{0}} .
$$

To demonstrate that (13) gives a good estimate for the shortest pulse achievable with a given fast saturable absorber, we carried out computer simulations of (1) with an absorber according to (4) and a fast absorber recovery time of only 50 fs. The other laser and absorber parameters are tabulated in Table I. The laser parameters model the experiments performed with a $\mathrm{Nd}$ :glass laser [12], to which we like to relate our simulation results later. All the numerical solutions of the master equation (1), shown in the following, are performed by the standard split-step-Fourier-transform method as discussed in Section II-B. The small-signal gain is varied from 0 to 0.2 in steps of 0.01 . The resulting stationary steady-state pulse intensities achieved after 20000 cavity round-trips without GDD and SPM are shown in Fig. 3. We clearly see, that as soon as the laser reaches threshold short pulses are formed. The pulses get shorter with increasing saturation of the absorber. Fig. 4 shows the pulsewidth and time-bandwidth product over the resulting intracavity pulse energy. We see already from the time-bandwidth product, that the resulting pulses are close to a sech and the pulsewidth is only slightly longer than the estimate given by (13) for increasing saturation of the absorber. The shortest pulse achieved in the simulation is 200 fs. For the parameters in Table I, we obtain from (13) for the shortest pulse $\tau_{\mathrm{FWHM}, \mathrm{FSA}}=170 \mathrm{fs}$, if we assume $g=l+q_{0}$. In this case, the resulting pulse series always results in a steady-state

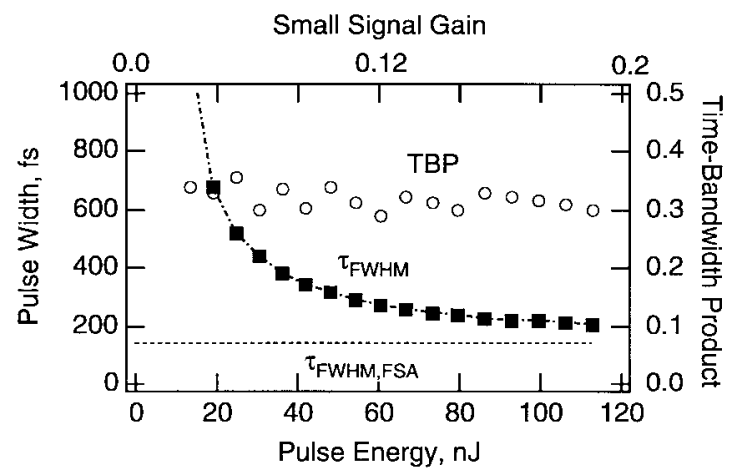

Fig. 4. The FWHM pulsewidth and the time-bandwidth products for the stationary pulses in Fig. 3 as a function of the intracavity pulse energy. The dashed line indicates the estimate for the shortest pulse achievable with an ideal fast saturable absorber according to (13).

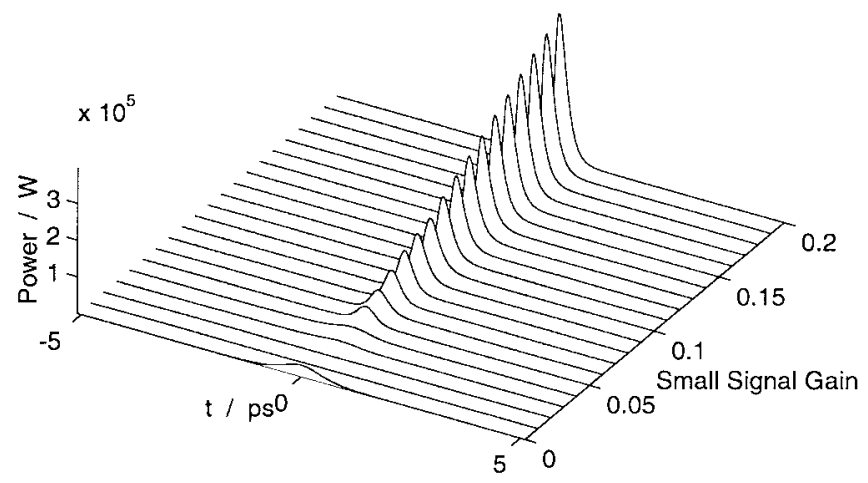

Fig. 5. Each trace shows the stationary pulse intensity profile obtained after 20000 cavity round-trips with an absorber response time $\tau_{A}=50 \mathrm{ps}$ for different values of the small-signal gain. The simulations are always started with a 1-ps initial pulse shown as the first trace.

single-pulse solution and the pulses do not break up, even if the absorber is further saturated. Thus, the simple formula (13) gives a good estimate for what can be expected for the pulsewidth given a certain amount of fast saturable absorption.

\section{B. Mode-Locking with a Slow Saturable Absorber}

If we keep all parameters constant and just increase the absorber recovery time from 50 fs to values as large as 10 ps, we find, surprisingly enough that the FWHM pulsewidth achievable with a given absorber does not increase very much. Fig. 5 shows the intensity profiles of the corresponding simulation results and Fig. 6 shows the pulsewidth and timebandwidth product over the pulse energy. We find, that for strong saturation of the absorber we can generate pulses that are only about $10 \%$ longer, i.e., $220 \mathrm{fs}$, as for those achieved with the fast saturable absorber that had a response time which was 200 times shorter. We obtain pulses which are about 30 times shorter than the absorber response time and do not show any instabilities on the scale of the simulation time of 20000 round-trips. We can explain this as follows. First, the filter loss decreases quadratically with the pulsewidth, (7). Thus, a pulse twice as long experiences only a fourth of the filter losses. Second, the absorber is now more strongly saturated than it has been before, because the response time is now much longer than the pulsewidth. The saturation energy of the absorber is 


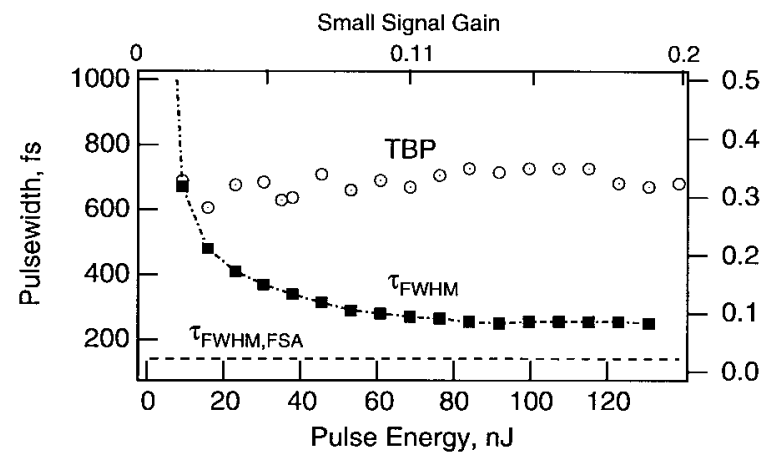

Fig. 6. The FWHM pulsewidth and the time-bandwidth products for the stationary pulses shown in Fig. 5 over the intracavity pulse energy. The top scale shows the corresponding small-signal gains.

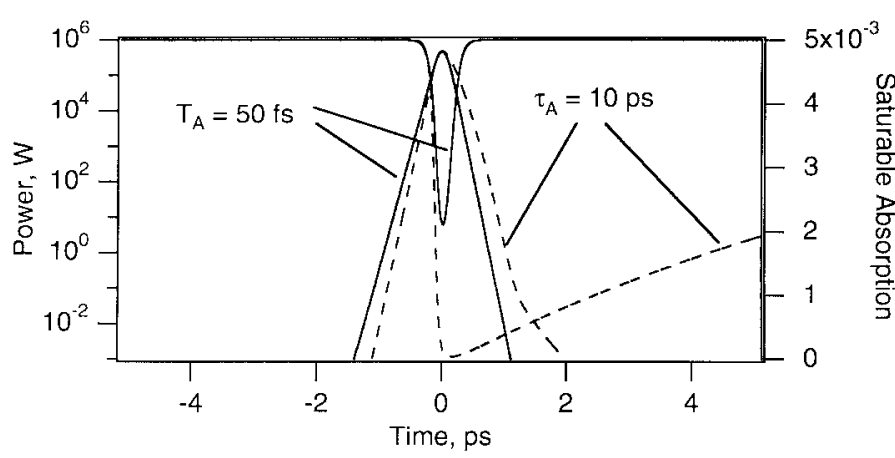

Fig. 7. Pulse power on a logarithmic scale and absorber response over time for a laser mode-locked with either a saturable absorber that has a 50-fs response time or a saturable absorber that has a 10-ps response time.

17 nJ, see Table I. For the largest pulse energies achieved, we saturate the absorber about eight times for the slow absorber, (Fig. 6). Thus the losses experienced by the pulse are much less than in the case of an ideal fast saturable absorber, i.e., the absorber is more strongly saturated. Therefore, the saturated gain level, extracted from the numerical simulation, saturates to a lower value, (Fig. 8), so that there is not too long a net gain window following the pulse. However, there is a prize to pay, the pulses develop a trailing wing due to the slowly recovering absorber, as can be seen from Fig. 7. Thus in terms of pulse quality and stability an absorber with a response as fast as possible is always desirable, as long as it can be sufficiently saturated with the available intracavity peak power, so that the saturable absorption can be exploited. We will show in the next section, that these limitations dissappear when soliton formation becomes the dominant pulse forming mechanism.

\section{Pulse Formation With Negative GDD AND SPM}

\section{A. The Fast Saturable Absorber}

In the case of the ideal fast saturable absorber without soliton-like pulse shaping, there is so much of the available saturable absorption reserved for stability, because the pulse has not only be kept stable by the saturable absorber, but also has to be shaped by the absorber. This behavior changes in the presence of negative GDD and SPM which has been studied in detail in [11]. The nondissipative, soliton-like pulse

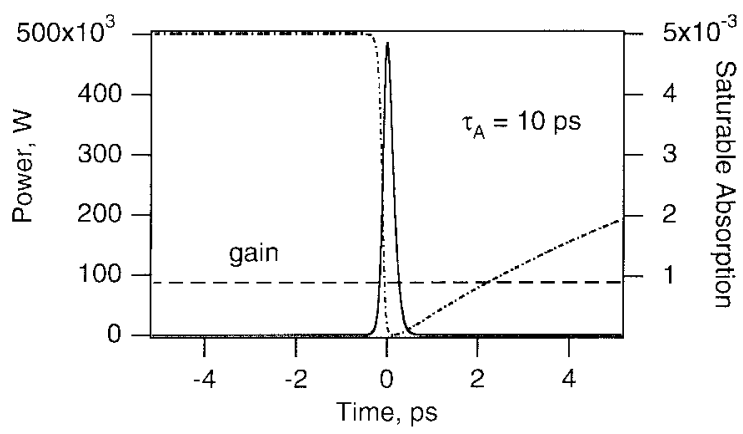

Fig. 8. Pulse power on a linear scale and absorber response together with the saturate gain level over time for a laser mode-locked with a saturable absorber response time of $10 \mathrm{ps}$.

shaping shapes already the pulse. The saturable absorber is then mainly responsible for the stabilization of this pulse. In the net negative dispersion regime, and for strong solitonlike pulse shaping, i.e., the SPM coefficient $\delta$ is much larger than the saturable absorption coefficient $\gamma$, the resulting exact solutions to the master equation (1) are very slightly chirped sech-pulses. Therefore, for this parameter regime an ideal soliton-like sech-pulse is still an excellent approximation to the exact solution. For an ideal soliton the relation between the pulsewidth and the pulse energy changes from (9) to

$$
\frac{|D|}{\tau^{2}}=\frac{1}{2} \delta\left|A_{0}\right|^{2} \quad \text { or } \quad \tau=\frac{4|D|}{\delta E_{P}} .
$$

The pulsewidth seems no longer to depend on the saturable absorption, which is of course not true. The finite gain bandwidth introduces additional loss to the soliton in comparison to a continuous-wave (CW)-signal that has a wavelength equal to the center wavelength of the gain. In each round-trip, the $\mathrm{CW}$-signal would experience more gain than the soliton and would break through. To avoid the CW-break through, a necessary condition is that the saturated gain level as shown in Fig. 2 stays below the loss level [11]. Then, we can compress the pulse to shorter durations than the width of the net gain window until all of the available saturable absorption that is reserved for pulse stability is used up to overcome the increasing filter losses. From Fig. 2 follows, that the minimum pulsewidth is reached when two thirds of the available saturable absorption above the unavoidable losses $q_{S}$ are used to overcome the losses in the gain medium due to the finite gain bandwidth

$$
\frac{D_{g, f}}{3 \tau^{2}}=\frac{2 q_{0}}{3} .
$$

The resulting minimum FWHM pulsewidth that can be achieved in this case is then twice as short as in (13)

$$
\tau_{\text {FWHM }}=\frac{1.76}{2} \cdot \sqrt{2 D_{g} / q_{0}} .
$$

For the laser parameters in Table I this minimum pulsewidth corresponds to $\tau_{\mathrm{FWHM}}=85 \mathrm{fs}$. Similar results have been obtained in [11]. However, as we will see later, there is another condition that has to be met which is stability against breakup into multiple pulses. 


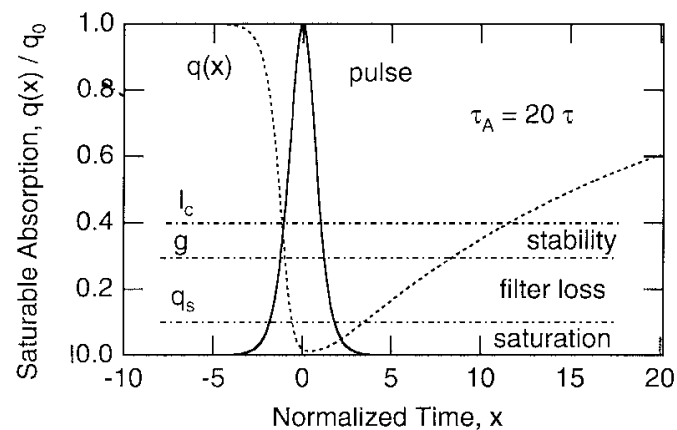

Fig. 9. Loss and gain dynamics for a secant hyperbolic pulse of a laser mode-locked by a slow saturable absorber and soliton formation.

\section{B. Mode-Locking with a Slow Saturable Absorber}

For a mode-locked laser with strong soliton-like pulse shaping, the minimum pulsewidth achievable with a given amount of saturable absorption, does not any longer rely on the creation of a window of net gain as short as the pulse itself. The pulse can be compressed below the width of the net gain window. In the fast saturable absorber case, this allows for an additional pulse shortening up to a factor of two given the same amount of saturable absorption, see (13) and (16). Of course, the same pulse shortening by a factor of two could be simply achieved by a saturable absorber four times as strong as before. However, a real saturable absorber also introduces a considerable amount of nonsaturable losses, which is usually proportional to the saturable absorption. Increasing the linear intracavity absorption reduces the efficiency of the laser and may even lead to such high losses that the laser cannot reach threshold. Thus, it is very important, that a saturable absorber with a given amount of saturable absorption can be used as efficiently as possible to generate the shortest pulses. The soliton-like pulse shaping present in most femtosecond solidstate lasers helps in this respect. Soliton formation does not only lead to shorter pulses in the case of a fast saturable absorber, it also leads to shorter pulses in the case of a slow saturable absorber as we have shown in [4]. In the case of a slow saturable absorber the reduction in pulsewidth can be even larger than a factor of two, because the pulse shaping due to the absorber is reduced.

The response of the slow absorber together with the saturated gain level and the total time dependent loss is shown in Fig. 9. The line $l_{c}$ shows the loss of a possible long background pulse, which is also called continuum in the language of soliton perturbation theory. Notice, the continuum loss is not any longer equal or close to the total linear intracavity losses as in the case of the fast saturable absorber. This is due to the fact that the slow absorber eventually leaves a long net gain window, following the pulse. In this net gain window the continuum can grow. However, the dispersion which is balanced for the soliton-like pulse by the SPM nonlinearity is not balanced for the continuum, i.e., the background pulse. Therefore, the continuum is spread into the regions of the slowly recovering loss, which leads to a cleanup of the continuum and a stabilization of the soliton-like pulse. The situation is shown in Fig. 9 for a pulse energy ten times the saturation energy of the absorber. For stability of the solitary pulse against the continuum it is required that the saturated gain has to be lower than the continuum loss $l_{c}$, so that the continuum does not reach threshold. Fig. 9 shows a net gain window following the pulse, which is about four times as long as the pulse itself. The situation shown could never be a stable situation in a purely dissipative mode-locking scheme. Therefore, we call this regime of mode-locking soliton-mode-locking. In a previous experiment, using $\mathrm{GaAs}-\mathrm{AlGaAs}$ quantum wells to realize a broad-band semiconductor saturable absorber on a silver mirror, we achieved pulses as short as $13 \mathrm{fs}$ at $820 \mathrm{~nm}$ from a standard Ti:sapphire laser. These pulses exist over the full stability range of the laser cavity and the laser is always self-starting in contrast to short pulse Kerr-lens mode-locked lasers. Computer simulations show, that if the SPM and GDD is switched off, the pulse widens to a width of about $40 \mathrm{fs}$, so that the net gain is zero immediately before and after the pulse, similar to what is shown in Fig. 2. Note, the pulsewidth shows a square root dependence on the absorber strength. Thus in order to achieve an additional pulse shortening by a factor of three, without using soliton-like pulse shaping, would necessitate an absorber roughly ten times stronger. In order to reach 10-fs pulses in a Ti:sapphire laser a few percent of saturable loss are already necessary. Thus, if we have to increase this amount by another factor of ten, the laser would not reach threshold anymore or it would run very inefficiently. This demonstrates the importance of the soliton-like pulse shaping to reach the shortest pulse with a given absorber strength.

However, stability against the continuum is not the only stability requirement. If we assume, that the pulse is completely shaped by the soliton-like pulse shaping process the FWHM pulsewidth is given from (14)

$$
\tau_{\mathrm{FWHM}}=1.76 \cdot \frac{4|D|}{\delta E_{P}}
$$

where $E_{P}$ denotes the pulse energy. With increasing pulse energy, of course the absorber becomes stronger saturated which leads to shorter pulses due to the saturable absorber and the soliton formula. At some point the absorber will saturate and can not provide any further pulse stabilization. However, the Kerr nonlinearity does not saturate and therefore the soliton formula dictates an ever decreasing pulsewidth for increasing pulse energy. This only works, until either the continuum breaks through, because the soliton loss becomes larger than the continuum loss, or the pulse breaks up into two pulses. The pulses will have reduced energy per pulse, such that each pulse is longer and experiences a reduced loss due to the finite-gain bandwidth. Due to the reduced pulse energy each of the pulses will suffer increased losses in the absorber, since it is not any longer as strongly saturated as before. However, once the absorber is already too strongly saturated by the single-pulse solution, it will also be strongly saturated for the double-pulse solutions, but the filter loss due to the finite-gain bandwidth are heavily reduced for the double-pulse solution. As a result, the pulse will break up into double pulses. To find the transition point where the breakup into multiple pulses occurs, we write 
down the round-trip loss due to the gain and filter losses and the saturable absorber according to (7)

$$
l_{m}=\frac{D_{g, f}}{3 \tau_{m}^{2}}+q_{S}\left(E_{P, m}\right)
$$

with the pulse energy $E_{P, m}$ of one pulse of the multiple pulse solution. The energy $E_{P, m}$ is determined from the total gain loss balance (7)

$$
\frac{g_{0}}{1+\frac{m \cdot E_{P, m}}{P_{L} T_{R}}}=l+l_{m} .
$$

Most often, the saturable absorber losses are much smaller than the losses due to the output coupler. In that case the total losses are fixed independent of the absorber saturation and the filter losses. Then the average power does not depend on the number of pulses in the cavity. If this is the case, one pulse of the double-pulse solution has about half of the energy of the single-pulse solution, e.g., $E_{P, 2}=\frac{1}{2} E_{P, 1}$ and, therefore, the width of the double pulse is twice as large as that of the singlepulse $\tau_{2}=2 \tau_{1}$ according to (17). Then the filter and absorber losses for the single- and double-pulse solution are given by

and

$$
l_{1}=\frac{D_{g, f}}{3 \tau_{1}^{2}}+q_{S}\left(E_{P, 1}\right)
$$

$$
l_{2}=\frac{D_{g, f}}{12 \tau_{1}^{2}}+q_{S}\left(\frac{E_{P, 1}}{2}\right)
$$

The single-pulse solution is stable against breakup into double pulses as long as

$$
l_{1}<l_{2}
$$

i.e., the difference in the filter losses between the single and double-pulse solution is smaller than the difference $\Delta q_{S}\left(E_{P, 1}\right)$ in the saturable absorber losses

$$
\frac{D_{g, f}}{4 \tau_{1}^{2}}<\Delta q_{S}\left(E_{P, 1}\right)=q_{S}\left(\frac{E_{P, 1}}{2}\right)-q_{S}\left(E_{P, 1}\right) .
$$

Fig. 10 shows the difference $\Delta q_{s}\left(E_{P}\right)$ in the saturable absorption for a single- and a double-pulse solution as a function of the ratio between the single-pulse peak power and saturation power for a fast absorber and as a function of the ratio between the single-pulse energy and saturation energy for a slow absorber. Thus, for both cases the optimum saturation ratio, where the largest discrimination between single and double pulses occurs and, therefore, where the shortest pulse can be expected before breakup into multiple pulses occurs is about 3. Note, to arrive at this absolute number, we assumed that the amount of saturable absorption is neglegible in comparison with the other intracavity losses, so that the gain dispersion is fixed. At this optimum operation point, the discrimination against pulse breakup with a fast absorber is about 50\% larger than the value of the slow absorber. Since the minimum pulsewidth scales with the square root of $\Delta q_{S}\left(E_{P}\right)$, see (22), the minimum pulsewidth of the slow absorber is only about $22 \%$ longer than with an equally strong fast saturable absorber. Fig. 10 also predicts that a

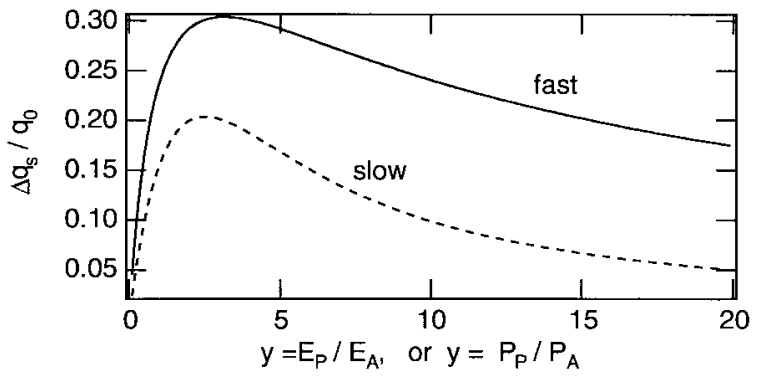

Fig. 10. Difference in loss experienced by a sech-shaped pulse in a slow (--) and a fast (-) saturable absorber for a given pulse energy $E_{P}$ or peak power $P_{P}$, respectively. $P_{A}$ is the saturation power of the fast absorber and $E_{A}$ the saturation energy of the slow absorber.

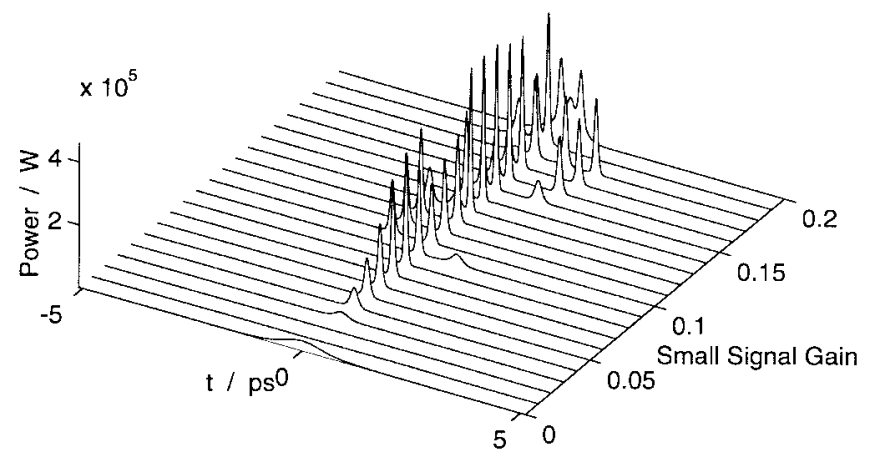

Fig. 11. Each trace shows the pulse intensity profile obtained after 20000 cavity round-trips in a diode-pumped $\mathrm{Nd}$ :glass laser according to [12] and Table I (with an absorber response time $\tau_{A}$ of $200 \mathrm{fs}$ ). When the laser reaches the double-pulse regime the multiple pulses are in constant motion with respect to each other. The resulting pulse train is not any longer stationary in any sense.

laser mode-locked by a fast saturable absorber is much more stable against multiple pulse breakup than a slow saturable absorber if it is oversaturated. This is due to the fact that the fast saturable absorber saturates with the peak power of the pulse in comparison with the slow saturable absorber which saturates with the pulse energy. When the pulse breaks up into a pulse twice as long with half energy in each, the peak power of the individual pulses changes by a factor of four. Therefore, the discrimination between long and short pulses is larger in the case of a fast saturable absorber, especially for strong saturation. Note, Fig. 10 is based on the simple saturation formulas for fast and slow saturable absorbers according to (3) and (4). We compare these predictions with numerical simulations and experimental observations made at the example of a $\mathrm{Nd}$ :glass laser [12].

The Nd:glass laser described in [12] was mode-locked by a saturable absorber which showed a fast recovery time of $\tau_{A}=200 \mathrm{fs}$, a modulation depth $q_{0}=0.005$, and a saturation energy $E_{A}=17 \mathrm{~nJ}$. The other laser parameters are those listed in Table I. Without the soliton-like pulse formation (GDD and SPM is switched off), the laser is predicted to produce about 200-fs short pulses with a single pulse per round-trip, very similar to what is shown for the fast saturable absorber in Fig. 3. The dynamics becomes completely different if the negative GDD and SPM are included in the simulation, (Fig. 11). With increasing small-signal gain, i.e., increasing pulse energy, the soliton shortens to $80 \mathrm{fs}$ due to the soliton- 


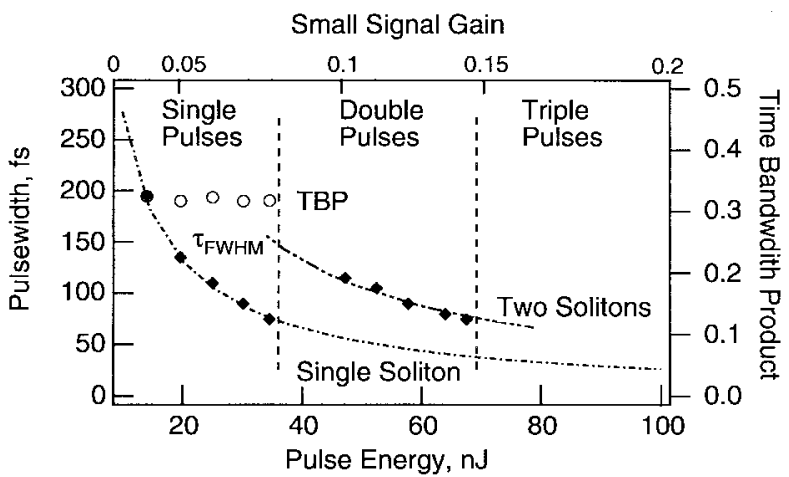

Fig. 12. Steady-state pulsewidth $(\checkmark)$ and time-bandwidth product (o) for a Nd:glass laser mode-locked by a saturable absorber with a 200 -fs recovery time with GDD and SPM included, shown as a function of the intracavity pulse energy. The time-bandwidth product is only meaningful in the single-pulse regime, where it is shown. The pulses are almost transform limited sech-pulses and follow the simple prediction of (17). The pulsewidth in the multiple pulseing regime is only unique in the parameter region where multiple pulses of similar height and width are achieved. The pulses break up into multiple pulses when the absorber is about three times saturated.

like pulse shaping, (Fig. 12). The pulsewidth follows nicely the soliton relation (17), dash-dotted line. The pulses become shorter, by about a factor of 2.5 , than without GDD and SPM before the pulse breaks up into longer double pulses. The pulse breakup into double pulses occurs, when the absorber is about two times saturated, close to the point where the shortest pulse can be expected according to the simple relations described above and visualized in Fig. 10. Fig. 11 shows, that the breakup point for the double pulses is also very close to the instability for continuum breakthrough. Indeed the first pulse train after breakup at a small-signal gain of $g_{0}=0.09$ shows the coexistance of a longer and a shorter pulse, which indicates continuum breakthrough. But the following five traces are double pulses of equal height and energy. For even stronger saturation of the absorber the double pulses break up into triple pulses and so on. Then the dynamics becomes even more complex. This behavior has been observed recently in a $\mathrm{Nd}$ :glass laser [12], see Fig. 13, as well as in $\mathrm{Cr}^{4+}:$ YAG lasers [13]. The simulations just discussed match the parameters of the Nd:glass experiments. Fig. 13 clearly shows the scaling of the observed pulsewidth according to the soliton formula until the pulses break up at a saturation ratio of about 2, which is close to the expected one of about 3 . The breakup into pure double and triple pulses can be observed more clearly if the absorber recovery time is chosen to be shorter, so that continuum breakthrough is avoided. Fig. 14 shows the final simulation results obtained after 20000 round-trips in the cavity, if we reduce the absorber recovery time from 200 to $100 \mathrm{fs}$, again for different small-signal gains, e.g., intracavity power levels and pulse energies. Now, we observe a clean breakup of the single-pulse solution into double-pulses and at even higher intracavity power levels the breakup into triple pulses without continuum generation in between. Note, the spacing between the pulses is very much different from what has been observed for the 200-fs response time. This spacing will depend on the details of the absorber and may also be influenced by the dynamic gain saturation, even if it is only a very small effect in this case [13].

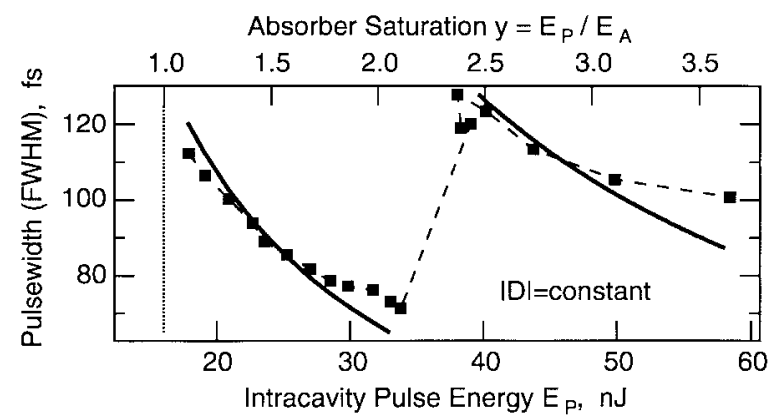

Fig. 13. Loss and gain dynamics for a secant hyperbolic pulse of a laser mode-locked by a slow saturable absorber and soliton formation.

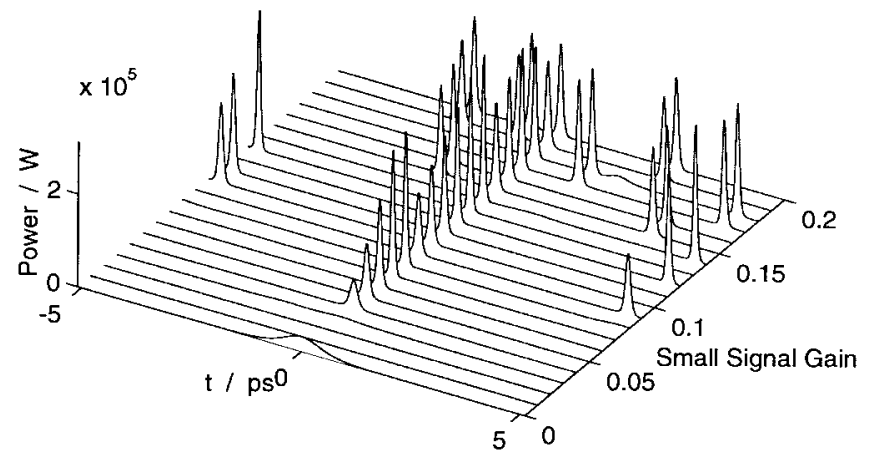

Fig. 14. Each trace shows the pulse intensity profile obtained after 20000 cavity round-trips for an absorber with a response time $T_{A}=100 \mathrm{fs}$ for different values of the small-signal gain. The simulations are always started with a 1-ps initial pulse shown as the first trace. Note, only the single-pulse solutions are stationary.

\section{INFLUENCE OF THE DISCRETENESS OF THE INTRACAVITY ELEMENTS}

So far, the numerical simulation of the master equation (1) by the split-step-Fourier-transform method has been performed by applying the net GDD per round-trip in one step and the net SPM per round-trip in one step according to the parameters listed in Table I. The master equation (1) assumes a continuous distribution of the GDD and SPM over one round-trip. In the real laser, the action of the GDD and SPM is discrete very much as in the split-step-Fourier transform. It is important to know at which power levels and pulsewidth the discrete action of the SPM and GDD leads to considerable deviations from the average model assumed in (1). One expects, that the discrete action of the SPM and GDD will lead to additional losses for shorter pulses in comparison to long pulses due to the higher peak power and increased spectral width. Therefore, the discrete nature of the two processes in the laser will also lead to a pulse breakup if the peak-phase shift due to the SPM and the pulse shaping due to the GDD becomes too large per round-trip. We show this SPM induced breakup for the $\mathrm{Nd}$ :glass laser model above by fixing the small-signal gain at a value of $g_{0}=0.06$, so that we stay below the breakup into multiple pulses due to the bandwidth limitations imposed by the gain. Then, we vary the SPM and the negative GDD linearly from $\delta=0.1 / \mathrm{MW}$ to $2 / \mathrm{MW}$ and $|D|=37.5$ to 750 $\mathrm{fs}^{2}$, respectively, such that the ratio between the SPM and GDD stays constant in each run, i.e., the pulsewidth of the 


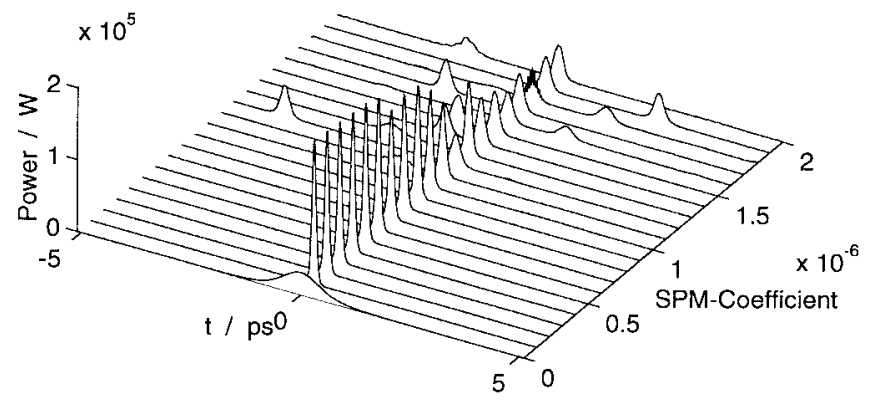

Fig. 15. Each trace shows the pulse intensity profile obtained after 20000 cavity round-trips for an absorber with a response time $T_{A}=200$ fs for different values of the SPM coefficient indicated on the left. The simulations are always started with a 1-ps initial pulse shown as the first trace. Note only the single-pulse solutions are stationary.

corresponding soliton should stay constant. Then the laser is simulated over 20000 round-trips and the resulting pulse trains are shown in Fig. 15. We see, that the pulse shape stays constant for SPM values greater than $\delta=0.2 / \mathrm{MW}$, when the soliton-like pulse shaping becomes strong enough. However, for SPM values as large as $\delta=1 / \mathrm{MW}$ the pulse shape starts to change again. The pulse broadens and a background occurs or the pulse breaks up as observed before. In total, we see for the given case that the deviation from the average dynamics described by (1) occurs when the nonlinear phase shift, e.g., the product of the peak pulse power and the SPM reaches about a value of $\Phi_{n l}=0.2$ which is more than two times larger then the nonlinear phase shift that occurs before the pulses break up in the simulations shown in Fig. 11. Nevertheless, the influences of the discreteness of the pulse shaping processes might already influence the dynamics of the $\mathrm{Nd}$ :glass laser in [12] beyond the first breakup point. In total, this discussion shows that the discrete action of the intracavity elements leads to additional losses for the pulse, which results in very similar effects as the finite gain bandwidth. In the case studied here this occurs for nonlinear phase shifts larger than $\Phi_{n l}=0.2$ per round-trip. As we have seen above, the pulse stability depends critically on the amount of saturable absorption employed, and how close we operate the laser at the threshold to instabilities where the minimum pulsewidth can be expected. Therefore, the precise value of the nonlinear phase shift where the discrete action of the SPM drives the pulse unstable or makes a visible difference to the average dynamics depends critically on the amount of saturable absorption in the laser which stabilizes the pulse. The effects of the discreteness of the elements is most pronounced in fiber lasers and the sub-10-fs Ti:sapphire lasers where the typical intracavity peak powers and/or the focussing into the gain material is stronger than in the case discussed here. However, it has to be noted that in the Nd:glass laser model used here we had only a saturable absorber with $0.5 \%$ modulation depth. In the sub10 -fs lasers, we typically had SESAM's with several percent of modulation depth [15] and the KLM induced modulation depth can be several ten percent. In that case much higher nonlinear phase shifts are necessary to drive the mode-locking process unstable.

\section{CONCLUSION}

We have shown that one can also employ slow saturable absorbers for short pulse generation. Already without any soliton-like pulse shaping, such an absorber can generate pulses much shorter than the recovery time of the absorber at the expense of some pulse broadening when compared with a fast saturable absorber of equal strength and similar saturation under pulsed excitation. If additional soliton-like pulse shaping is present, the pulse can be further compressed below the width of the net gain window and further shortened by a factor of about two in the case of a fast saturable absorber and even more in the case of a slow saturable absorber. The soliton-like pulse shaping, which decouples to a large extend the pulse shaping from the stabilization that is provided by the saturable absorber, may lead to a breakup of the single pulse per round-trip into many pulses per round-trip. The most stable operation against breakup into multiple pulses is obtained, if the absorber is about three times saturated (i.e., the intracavity pulse energy is about three times the saturation energy of the absorber). A laser mode-locked by a fast saturable absorber is more stable against multiple pulse breakup than a slow saturable absorber, especially if it is oversaturated. One has to be aware, that the saturation behavior of a real semiconductor saturable absorber can be more complex than the simple saturation formulas derived for a two-level system in the limit of a fast or slow recovery time. Nevertheless, the formulas and operation guidelines derived here in using fast or slow saturable absorbers for the mode-locking of lasers allow for a design of the absorber for the power levels and pulsewidth one wants to achieve with a given system. The general arguments presented are true even if the saturation characteristics of the absorber deviates from the simple relations for a two-level system. For example, the saturation characteristics of a semiconductor absorber will definitely depend on the pulsewidth as well as on the excitation wavelength. SESAM's offer the additional benefit that the detailed saturation charcteristics of the absorber can be determined by independent pump-probe measurements [2], [15]. From the measured saturation characteristics, one can compute the new optimum operation point according to (22) for the given absorber.

\section{ACKNOWLEDGMENT}

The authors thank C. Hönninger for characterization of the time constants of the semiconductor absorbers used in the $\mathrm{Nd}$ :glass experiments.

\section{REFERENCES}

[1] U. Keller, D. A. B. Miller, G. D. Boyd, T. H. Chiu, J. F. Ferguson, and M. T. Asom, "Solid-state low-loss intracavity saturable absorber for Nd:YLF lasers: An antiresonant semiconductor Fabry-Perot saturable absorber," Opt. Lett., vol. 17, pp. 505-507, 1992.

[2] U. Keller, K. J. Weingarten, F. X. Kärtner, D. Kopf, B. Braun, I. D. Jung, R. Fluck, C. Hönninger, N. Matuschek, and J. Aus der Au, "Semiconductor saturable absorber mirros (SESAM's) for femtosecond to nanosecond pulse generation in solid-state lasers," IEEE J. Select. Topics Quantum Electron., vol. 2, pp. 435-453, 1996. 
[3] U. Keller, "Semiconductor nonlinearities for solid-state laser modelocking and $Q$-switching," in Nonlinear Optics in Semiconductors, A. Kost and E. Garmire, Eds. Boston, MA: Academic, 1998.

[4] F. X. Kärtner and U. Keller, "Stabilization of soliton-like pulses with a slow saturable absorber," Opt. Lett., vol. 20, pp. 16-18, 1995.

[5] F. X. Kärtner, I. D. Jung, and U. Keller, "Soliton modelocking with saturable absorbers," IEEE J. Select. Topics Quantum Electron., vol. 2, pp. 540-556, 1996.

[6] I. D. Jung, F. X. Kärtner, L. R. Brovelli, M. Kamp, and U. Keller, "Experimental verification of soliton modelocking using only a slow saturable absorber," Opt. Lett., vol. 20, pp. 1892-1894, 1995.

[7] O. E. Martinez, R. L. Fork, and J. P. Gordon, "Theory of passively modelocked lasers including self-phase modulation and group-velocity dispersion," Opt. Lett., vol. 9, pp. 156-158, 1984.

[8] _ "Theory of passively modelocked lasers for the case of a nonlinear complex propagation coefficient," Opt. Soc. Amer. B, vol. 2, p. 753,1985

[9] F. Salin, P. Grangier, G. Roger, and A. Brun, "Observation of highorder solitons directly produced by a femtosecond ring laser," Phys. Rev. Lett., vol. 56, pp. 1132-1135, 1986.

[10] _ "Experimental observation of nonsymmetrical $N=2$ solitons in a femtosecond laser," Phys. Rev. Lett., vol. 60, pp. 569-571, 1988.

[11] H. A. Haus, J. G. Fujimoto, and E. P. Ippen, "Structures for additive pulse modelocking," Opt. Soc. Amer. B, vol. 8, pp. 2068-2076, 1991.

[12] J. Aus der Au, D. Kopf, F. Morier-Genoud, M. Moser, and U. Keller, "60-fs pulses from a diode-pumped Nd:glass laser," Opt. Lett., vol. 22, pp. 307-309, 1997.

[13] B. C. Collings, K. Bergman, and W. H. Knox, "Truely fundamental solitons in a passively mode-locked short cavity $\mathrm{Cr}^{4+}$ :YAG laser," Opt. Lett., vol. 22, pp. 1098-1100, 1997.

[14] R. Fluck, I. D. Jung, G. Zhang, F. X. Kärtner, and U. Keller, "Broadband saturable absorber for $10 \mathrm{fs}$ pulse generation," Opt. Lett., vol. 21, pp. 743-745, 1996.

[15] I. D. Jung, F. X. Kärtner, N. Matuschek, D. H. Sutter, F. Morier-Genoud, Z. Shi, V. Scheuer, M. Tilsch, T. Tschudi, and U. Keller, "Semiconductor saturable absorber mirrors supporting sub-10 fs pulses," Appl. Phys. B (special issue on ultrashort pulse generation), vol. 65, pp. 137-150, 1997.

[16] I. D. Jung, F. X. Kärtner, N. Matuschek, D. H. Sutter, F. Morier-Genoud, G. Zhang, U. Keller, V. Scheuer, M. Tilsch, T. Tschudi, "Self-starting 6.5 fs from a Ti:sapphire laser," Opt. Lett., vol. 22, pp. 1009-1011, 1997.

[17] H. A. Haus, "Theory of modelocking with a fast saturable absorber," $J$. Appl. Phys., vol. 46, pp. 3049-3058, 1975.

[18] C. Spielmann, P. F. Curley, T. Brabec, and F. Krausz, "Ultrabroadband femtosecond lasers," IEEE J. Quantum Electron., vol. 30, pp. 1100-1114, 1994

[19] I. P. Christov, H. C. Kapteyn, M. M. Murnane, C. P. Huang, and J. Zhou, "Space-time focusing of femtosecond pulses in a Ti:sapphire laser," Opt. Lett., vol. 20, pp. 309-311, 1995.

[20] I. P. Christov, V. D. Stoev, M. M. Murnane, and H. C. Kapteyn, "Sub10-fs operation of Kerr-lens mode-locked lasers," Opt. Lett., vol. 21, pp. 1493-1495, 1996.

[21] K. Tamura, E. P. Ippen, H. A. Haus, and L. E. Nelson, "77-fs pulse generation from a stretched-pulse modelocked all-fiber ring laser," Opt. Lett., vol. 18, pp. 1080-1082, 1993.

[22] H. A. Haus, K. Tamura, L. E. Nelson, and E. P. Ippen, "Strechtedpulse additive pulse mode-locking in fiber ring lasers: Theory and experiment," IEEE J. Quantum Electron., vol. 31, pp. 591-598, 1995.

[23] H. A. Haus, "Parameter ranges for cw passive modelocking," IEEE $J$. Quantum Electron., vol. QE-12, pp. 169-176, 1976.

[24] F. X. Kärtner, L. R. Brovelli, D. Kopf, M. Kamp, I. Calasso, and U. Keller, "Control of solid-state laser dynamics by semiconductor devices," Opt. Eng., vol. 34, pp. 2024-2036, 1995.

[25] G. P. Agrawal, Nonlinear Fiber Optics. New York: Academic, 1989.

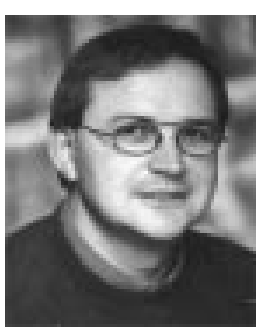

Franz X. Kärtner was born in Cham, Germany, in 1961. He received the Diploma degree in electrical engineering from the Technical University, Munich, Germany, in 1986 and the Ph.D. degree on noise in microwave circuits and optical devices in 1989

From 1991 to 1993, he was a Feodor-Lynen Research Fellow of the Humboldt Foundation at Massachusetts Institute of Technology, Cambridge, MA, where he carried out research on the generation of squeezed states in fibers and quantum nondemolition measurements. From 1993 to 1997, he was with the Institute of Quantum Electronics, Swiss Federal Institute of Technology $($ ETH), Zurich, Switzerland. He received his Habilitation in 1997 with work on ultrashort pulse generation. Since 1998, he is a Visiting Assistant Professor at the Massachusetts Institute of Technology.

Dr. Kärtner is a member of the German Physical Society and the Optical Society of America.

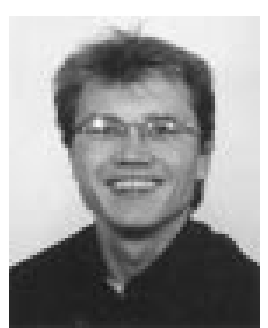

Juerg Aus der Au received the diploma degree in physics from the Swiss Federal Institute of Technology (ETH) in Zürich, Switzerland, in 1996 and is working towards the Ph.D. degree at the same institute, where his main research interests are focused on compact high-power femtosecond laser sources.

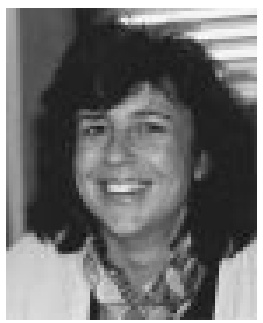

Ursula Keller (M'89) was born in Zug, Switzerland, in June 1959. She received the "Diplom" in physics from the Federal Institute of Technology (ETH), Zurich, Switzerland, in 1984, and the M.S and $\mathrm{Ph} . \mathrm{D}$. degrees in applied physics from Stanford University, Stanford, CA, in 1987 and 1989 respectively.

Her Ph.D. research was in optical probing of charge and voltage in GaAs integrated circuits and in low-noise ultrafast laser systems. From late 1984 to 1985 , she worked on optical bistability at HeriotWatt University, Edinburgh, Scotland, U.K. In 1989, she joined AT\&T Bell Laboratories, Holmdel, NJ, as a Member of Technical Staff where she conducted research on photonic switching, ultrafast laser systems, and semiconductor spectroscopy. Since March 1993, she has been an Associate Professor and since October 1997, a Full Professor in the Physics Department at the Swiss Federal Institute of Technology (ETH), Zurich, Switzerland. Her current research interests are in ultrafast lasers, spectroscopy, local probes and novel devices for applications in optical information processing, communication and medicine. She published more than 80 journal papers, one book chapter and holds five patents.

Prof. Keller is a member of the Optical Society of America (OSA), the European Physical Society (EPS), the Swiss Physical Society (SPS), and the Swiss Academy of Technical Sciences (SATW). During 1985-1986, she was a Fulbright Fellow, in 1987-1988, she received an IBM Predoctoral Fellowship and in 1998 the Carl Zeiss Research Award. 